\title{
Research on the Virtual Access Points in the Wireless Local Area Network
}

\author{
Min Chen*, Zejun Li and Ang Li
}

School of Computer Science and Technology, Hunan Institute of Technology, Hengyang, China

\begin{abstract}
The management of the access points in the establishment of the current wireless local area network is independent and scattered. The builder must log into the access points with the artificial method to set the identification, the operating channel and the authentication code. The client stations have to scan each channel for finding out the pre-set identification and then cooperating the process of the authentication for the access network. These processes may take several seconds and greatly degrade the Internet experience, especially for the users who frequently move among different coverage of access points. Additionally, if users desire high throughput and ubiquitous network accessed by distributing high-density access points, the performance of whole network does not grow as expected due to the ill management of independent access points. In this paper, we extend the software-defined network (SDN) into the access network so that access points are managed by a central controller to deal with the issues, such as load balance, interference of channel allocation and handover. After the users can register an account and select the configuration for the server in advance, the configuration of the group can be directly adopted to enter the network. The server can create its special virtual access points in the neighboring access points, and then directly provide the service of the client side. When the client side moves, the virtual access points will move into other tangible access points with the moving of the client sides. As to the client sides, it has the same access points connection without observing whether the procedure of changing the hand exists.
\end{abstract}

Keywords: Software-defined network (SDN), virtual access points (VAP), wireless local area network (WLAN).

\section{INTRODUCTION}

With the popular of the personal mobile equipment and the diversified equipment of the enterprise, the requests of the wireless access network service is largely increased, such as the large-scale vocal concert, the exhibition place, and the meeting hall. The 5G METIS researching program in Europe proposes the following possible useful case. A four-day open-air festival is hold in the rural area with less than a square kilometer, and the activity can have at least 100,000 accesses and highly use the wireless network equipment. For example, the tourists hope that the recorded Hd video films can be uploaded to the Internet and then the share them with the friends and the family. In addition, the employees to support the normal activities, security personnel, medical personnel and related equipment can rely on the reliable wireless communication and the network access service.

The present wireless access network is still not mature enough to support the high density and a large number of network access. The mobile communication network in the next generation $(5 \mathrm{G})$ will attempt to integrate many different kinds of Multiple Radio Access Technologies, especially the mainstream technology of the Wireless Local Area Network in the computer communication field, digital home and office. The problem of the gaps and the blind angles can be offset in the traditional cellular mobile network through the integration of the wireless local network. In the meantime, the flow of the data packets can be shared so that a cheaper and faster data network service can be reached [1].
The wireless local network is initially designed to be used by the house or office and other private small-scale places. After an individual sets the name for the purchased access points devices, operating the channels and authenticating the code, the network can be operated on line. The deployment method of the present wireless local network adopts to the distributed self-management. Each access point should be set through the artificial logging. The neighboring access points cannot reach to the elf-adaptive access network. Therefore, when the deployment density of the access points is increasing, the problems appeared in the wireless local network are as follows: the speed of the online is slow or the online cannot be operated, the lines are often broken during the moving, and the online among many access points is often automatically selected. The network problems are as follows: 1 . The problem of the load balance. The wireless local network cannot distribute which access point should be used for the client side from the network terminal, and it should be selected by the strength of the signals. Therefore, the heavy access points load of the high-strength signal can be caused so that the networks are often broken or the speed of the network is rather slow. 2. The problem of the channel inference. When the access points are deployed, its operating channels are often set according to the preferences of the builders or random. While the network terminal cannot automatically set the operating channels in terms of the frequency environments. Therefore, the sharing channels of the access points and the mutual inferences can reduce the speed of the network. 3. The problem of the handover. There is the border limitation between the physical layer and the logical layer among the wireless local network access points, the access points can be individually operated. If the client sides 
wants to $\log$ in the new access point and then use the network, it must be scanned, authenticated and combined so that the reason of breaking up the online during the process of the handover cannot be simplified.

In order to improve the efficiency of the high-density wireless local network, and regard the $5 \mathrm{G}$ mobile communication multi access technology as the paving integration, the paper extends the Software-Defined Network to the wireless local network, as shown in the Fig. (1). The access points of the wireless local network can be managed in the central server through the network interface. The central server can reduce many problems of the above high-density wireless local network through the establishment of the Virtual migration.

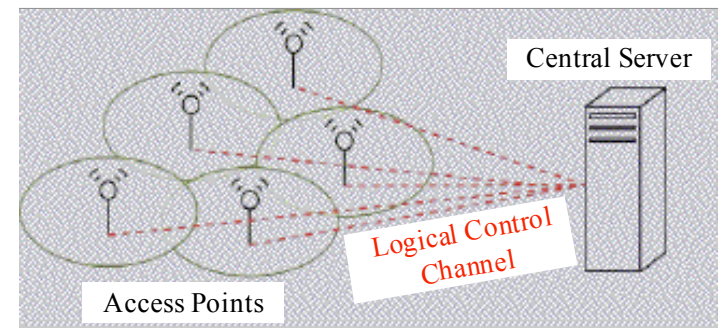

Fig. (1). The central server controls the operation of the actual access points through the logical channel, and then creates the service of the virtual access point.

\section{THE ESTABLISHMENT OF THE VIRTUAL AC- CESS POINTS}

If an area is densely covered overlap by many numbers of the access points, these access points are controlled by a central server through the network socket. For example, the server can control the channels operated in the access points, the broadcast contents of the beacon frame, the process of the authentication and the procedure of the association. The following contents will explain how we repeatedly adopt the existing IEEE 802.11 standard frame [2] to realize the handon operation of the virtual access points.

\subsection{The Register of Users}

The client side should register the willing used Service Set Identification in the central server, exchanging the authenticated keys and offering the client side's MAC address as the binding. In addition, the server can also regard the device capacity of the client side and preference settings as the references of establishing the personal virtual access points, such as the modulating code rate, the characteristics of the data flow (whether the voice, the video streaming is important or the web browsing is important) and the guarantee requirements of Quality of Service. In addition, the server adopts the information (SSID, MAC address) provided by the client side to produce a group of Basic Service Set Identifier which is regarded as the used MAC address during the process of establishing the virtual access points. The Basic Service Set Identifier can also notice the client side in the phase of the register so that it can be adopted in the process of connecting the network in the future.

\subsection{The Active Scanning and the Dynamic Establishment}

The IEEE 802.11 standard does not only provide the passive scanning, but also the active scanning. How to improve the establishing efficiency of the virtual access points through the active scanning will be discussed in the following contents. The client side does not passively listen to the beacon frame sent by the access points at all, actively asking the questions. The process of inquiry is finished by the Probe Request frame and the Probe Reply frame. The Probe Request frame is delivered by the client side, and the frame should be with the users' willing probe Service Set Identification, and it should be judged whether the access points of the received probe frame us the accessible Service Set Identification. If it is, the Probe Reply Frame should be delivered. After the Probe Reply Frame is received, the client side can obtain the access point corresponding the Service Set Identification.

In order to reduce the resources waste during the process of establishing the access points, the program of the active scanning is modified, as shown in the Fig. (2). The client side has been with the registered Service Set Identification and the Device Identification Code (STA_ID, such as MAC address), while the access points cannot automatically judge whether the Service Set Identification is effective after receiving the probe frame so that it should ask the latter central server. The method of the inquiry is to deliver the VAP Setup Request to the central server and then it should be with the AP_ID, the client side identification code and the requested Service Set Identification. After the central server receives the requests, it should judge whether the client side identification code and the Service Set Identification are the effective requests. In addition, the STA_profile of the users and the state of the physical access points required to deliver the requests determine whether the establishment of the virtual access points is checked. If the central server determines to establish the virtual access points, the VAP Setup Response should be done. The related parameters of the client side can be carried in the response so that the hand-over program of the subsequent client side and the virtual pints can be saved. After the physical access points receive the response from the central server, the corresponding virtual access points should be established according to the given preference settings from the client side. Later, the client side can conduct the following steps similar to the general physical access points: the authentication, the connection and the data transmission.

\section{THE MANAGEMENT OF THE ACCESS POINTS AND THE DISTRIBUTION OF THE CHANNELS}

It is assumed that the client side can passively receive the broadcast beacon frame from the access points or the access points can receive the probe frame actively delivered by the client side in the Chapter 2. In fact, the physical layer in the IEEE 802.11 can support the operations of different channels. When the client side and the physical access points are operated in different channels, they cannot receive and transmit the data in each other. In other words, the client side or the physical access points cannot get to know the existence of each other through the beacon frame or the probe frame. In this way, the establishment of the virtual access points cannot be conducted. The following contents will continue to discuss the distribution of the channels and the problem of the deployment foe the physical access points. 


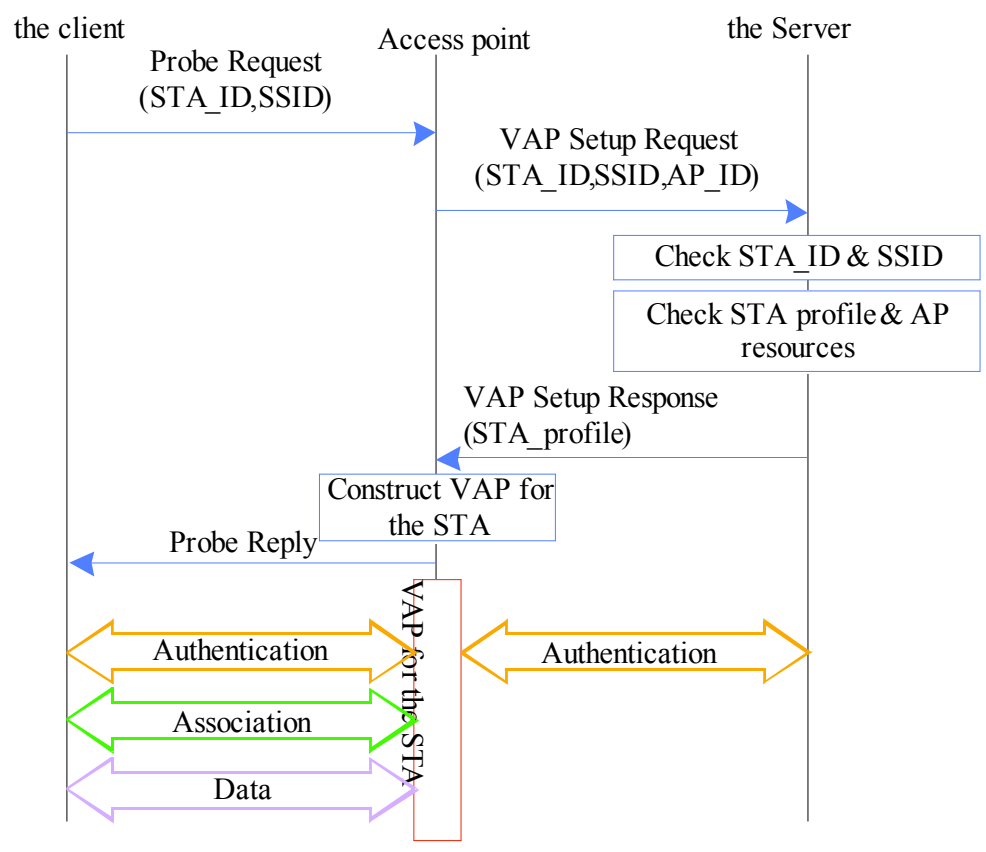

Fig. (2). The probe request frame is adopted to trigger the server for establishing the virtual access points.

\subsection{The Setting of the Access Points with the Same Fre- quency}

In order to avoid the client side not getting to know the operating channels of each physical access points, each possible communicating channel should be scanned. A kind of simple channel planning method is to make all physical access points operate in the same channel. In this way, when the client side registers in the central service, the server can directly notice the client side a fixed channel. Later, when the client side wants to establish a virtual access point to use the network service, the probe requests can be delivered to the virtual access points to the physical access points in the communicating channels (the procedure is as shown in the Fig. 2) without paying attention to the communication channels of the physical channel in its local area. In addition, the setting method of the same channel can also help the movement and the hand-over of the users. When the client side moves to the coverage range accessed from the different entities, the client side can save the time of switching and scanning among different channels and speeding up the procedure of the hand over by using the same communicating channel. Although the physical access points with the same frequency settings can bring the above benefits, while the problem of the same frequency inference should be solved. The method of the inference resistance is to adjust the operating parameters of the virtual access points through the servers, such as the beam-forming technology [3], the choice of the channel encoding [4] or the control of transmitting power [5]. The above mechanism of the inference resistance needs the state of the client side and the access points obtained from the servers and then make a decision through the proper deciding mechanism so that the access points and the client side should be conducted [6].

The Fig. (3) explains a set of mechanism of the state return and the state response. The central server requires the virtual access points to collect the related information of the client side online through the Status Request. If the virtual access points have no related information available for return, the client side should be required to measure the needed information through the Measurement Request. The 8.4.2.23 chapter specifies the related forms required by the measurement in the specification of the IEEE 802.11, as shown in the Fig. (4). The Measurement Type defines 12 kinds of types in total. For example, the type 2 and the type 4 can transmit back the Receive Power Indication and the statistical sheet of the size of the noise respectively, the type 3 can report back the number of the using load in the communicating channels, the type 7 can list the data, the encoding error of the control frame, the times of the loss and re-transmission. All of these data can assist the server to analyze the inference degree of the channels. When the client side finishes the related measurements and reports, the access points can collect the data themselves by reporting the status to the central server. After the server receives the status report, the state information can be adopted to do the decision, as shown in the Fig. (3). The procedure of the decision or the algorithm depend on the target of optimizing the network and the limited condition. All of these have been deeply discussed in related researches. The result of the decision is to deliver the Reconfiguration information to the access points through the server. The access points should be deployed and adjusted again. After it is finished, the actual Reconfiguration Conform should be reported back to the server, If the client side has something be adjusted in the reconfiguration information, the access points should deliver the related information to the client side [7-9].

\subsection{The Setting of the Access Points with the Different Frequencies}

The adjustment of the freedom degree of the communication interrupted problems from the exterior and the interior in the setting of the access points in the different frequencies. 


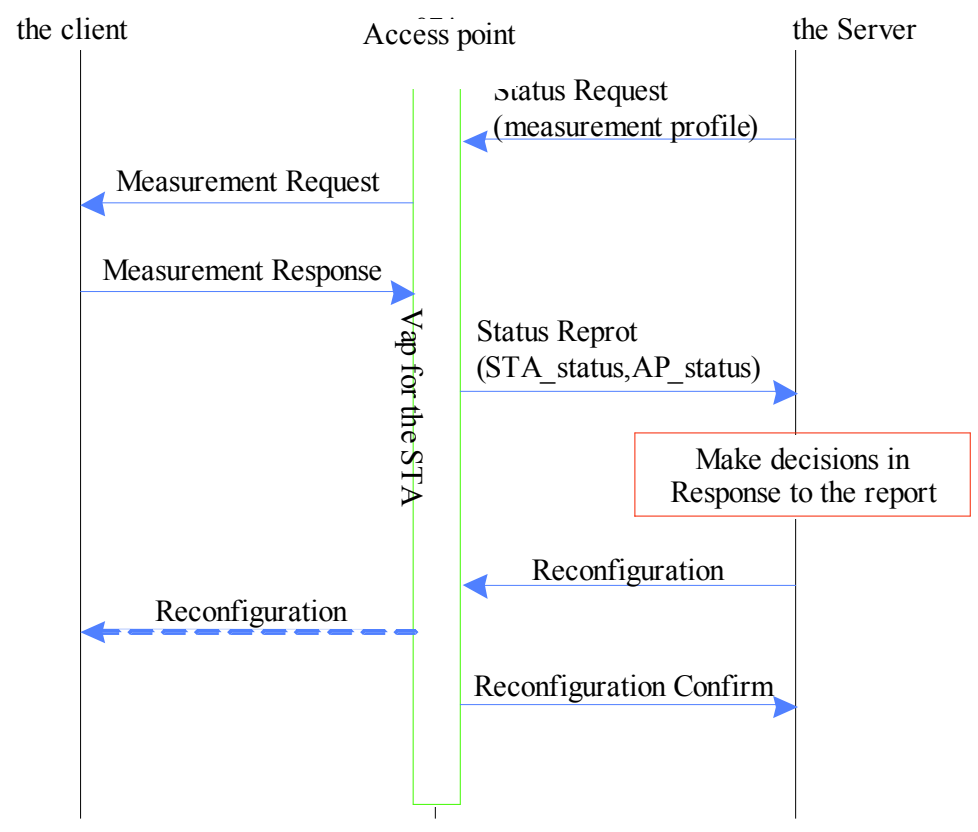

Fig. (3). The central server sends the state and returns the request, using the returned state to adjust the configuration of the virtual access points.

\begin{tabular}{|c|c|c|c|c|c|}
\hline $\begin{array}{c}\text { Element ID } \\
(1 \text { byte })\end{array}$ & $\begin{array}{c}\text { Length } \\
(1 \text { byte })\end{array}$ & $\begin{array}{c}\text { Measurement token } \\
(1 \text { byte })\end{array}$ & $\begin{array}{c}\text { Measurement Request Mode } \\
(1 \text { byte })\end{array}$ & $\begin{array}{c}\text { Measurement Type } \\
(1 \text { byte })\end{array}$ & $\begin{array}{c}\text { Measurement Request } \\
(\text { Variable })\end{array}$ \\
\hline
\end{tabular}

Fig. (4). The measurement request form delivered by the virtual access points to the client side.

For example, the neighboring access points can adopt to the different channels, and the channels interrupted by the exterior can be temporarily tripped off. With the different positions of the client side, the physical access points providing the service can also have different communication channels in the setting of the access points with the different frequencies. The client side can send the probe requests in the possible channels and then should be waited for the responses (as shown in the Fig. 2) until the correct channels are found out so that the responses can be received and the service of the virtual access points can be established. Therefore, how to reduce the delay of establishing the access points is one of the important discussions in the chapter [10-13].

The mechanism which does not need to the client side's channel scanning and still remains the access points with different frequencies is proposed. Firstly, when the client side registers in the central server, the server can notice the client side a fixed available communication channel and the general service identification code just the same as the access points with the same frequency. The channel is called as the control channel in the system, which is just adopted to the available channel during the phase of establishing the virtual access points. The establishing procedure of the access points with different frequencies is as shown in the Fig. (5). The physical access points can send the beacon frame in the control channel periodically with the general service identification code. When the client side wants to obtain the network service, the beacon frame in the control channel should be hear. When the service identification code is found out, the available physical access points can be ensured to locate in the neighboring places. The client side can actively initi- ates the probe request to trigger the establishing procedure of the virtual access points. The access points can send the establishing requests of the virtual access points to the server, in which the communication channels available to transmit the data can be searched out to the server. After the distributed determination of the channels, the server should assign the operating channels of the virtual access points in the request of replying the access points. The access points should establish the virtual access points in the original control channel. After the authentication and connection, the operating channels can be switched. The switching method of the operating channels should be conducted in the Channel Switch Announcement through the virtual access points. The 8.4.2.21 chapter in the IEEE 802.11 standard defines the form of the Channel Switch Announcement, as shown in the Fig. (6), in which the fourth segment represents the target channel of the switch, the fifth column notices the time of switching the channels after the number of the beacon frames are delivered. The use of the Channel Switch Announcement can make the virtual access points be operated from the control channels to the specified channels. Later, the client side can begin to transmit the data with the virtual access points and cannot cause the same frequency interruption by sharing the channel with other neighboring access points $[14,15]$.

A physical access point may need to serve multi client sides so that multi virtual access points should be established. These virtual access points should share with the resources of the physical access point each other. When many virtual access points with different frequencies should be operated in the same physical access point, the physical ac- 


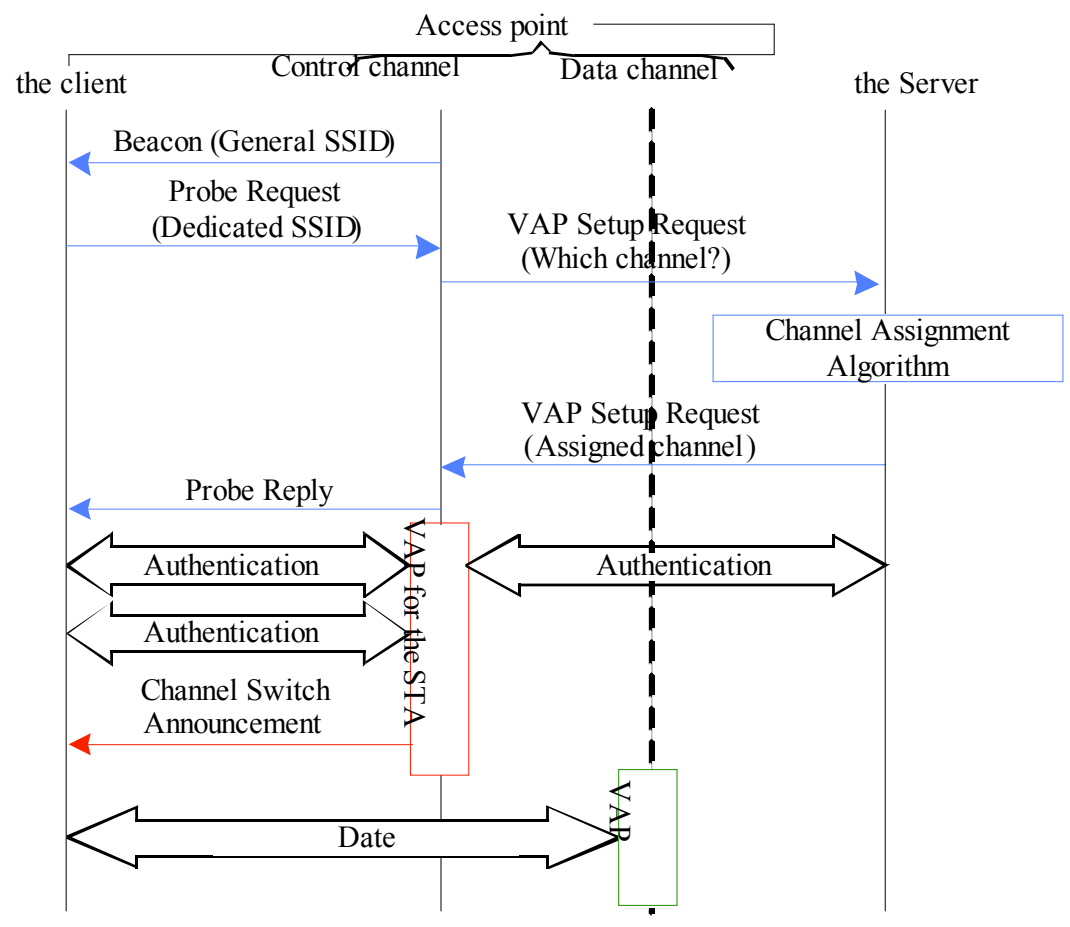

Fig. (5). The Mechanism of Establishing the virtual access points with different frequencies under the condition of not scanning the channels in the client side.

\begin{tabular}{|c|c|c|c|c|}
\hline $\begin{array}{c}\text { Element ID } \\
(1 \text { byte })\end{array}$ & $\begin{array}{c}\text { Length } \\
(1 \text { byte })\end{array}$ & $\begin{array}{c}\text { New Channel Number } \\
\text { Channel Switch Count }\end{array}$ \\
$(1$ byte $)$ & $(1$ byte $)$ \\
\hline
\end{tabular}

Fig. (6). The channel switch announcement format delivered by the virtual access points to the client side.

cess point should do the channel switching. When the physical access point is switched into other channels serving other client sides, the virtual access points operated in the original channel should be stopped so that the data transmitted in the client side cannot be received. The access points can do the communication in other channels, the client side cannot find out the channel located in the busy state through the carrier wave detecting mechanism of the original IEEE 802.11. Therefore, the client side can do the data transmission and cause the loss of the data.

In order to avoid the loss of the transmitted data in the client side, the mechanism with the virtual carrier wave detection is proposed so that the client side thinks the virtual access points are receiving the data from other client sides and the opportunity of using the channels can be delayed. The Fig. (7) explains the detail operating method of the mechanism. If there are two virtual access points respectively operated in the different channels in a physical access point, the access points should periodically send the new users of the beacon frame service to the control channels. Therefore, the access points must be switched in the three different channels. The access point which is used as the identification of the virtual access point $\mathrm{A}$ should do the data transmission between the channel $\mathrm{A}$ and the client side $\mathrm{A}$. When the access point wants to switch into the channel $\mathrm{B}$ for serving the client side $\mathrm{B}$, the virtual access point $\mathrm{A}$ can transmit the frame of the CTS-to-self. The CTS frame is the transmission of thee data frame by the receiving terminal notices the transmitting terminal. In the mean time, the neighboring other nodes is also noticed that it is receiving the data without using the channel which is located in the busy state. The time of the busy channels is noticed by the Network Allocation Vector allowed to sent the frame. Apart from the transmitting terminal, the nodes allowed to send the frames, the timer should be turned on and any transmissions should be stopped in the time. After the time is reached, the using right of the competition channel should be restarted. The CTS-to-self frame is to write itself address in the receiving address of the frame. Therefore, when the virtual access point A transmits CTS-to-self frame, the corresponding client side will stop using the channels in the given time. Later, the physical access point can safely switch into the channel $\mathrm{B}$ for serving the client site. Meantime,. when the virtual access point $\mathrm{B}$ transmits CTS-to-self frame, the physical access point can safely switch into other channels. For example, when the beacon frame is delivered in the control channel, the connection of the new client side should be awaited. The access point can be switched in different channels and not loss the information of receiving the client side with the use of the CTS-to-self frame.

In addition, the time ratio of distribution in each channel by the physical access points should be distributed by the central service for increasing the whole frequency using efficiency of the network. The method is to send the Scheduling Request to the central service through the virtual access points. The expected using number of the channels and the 


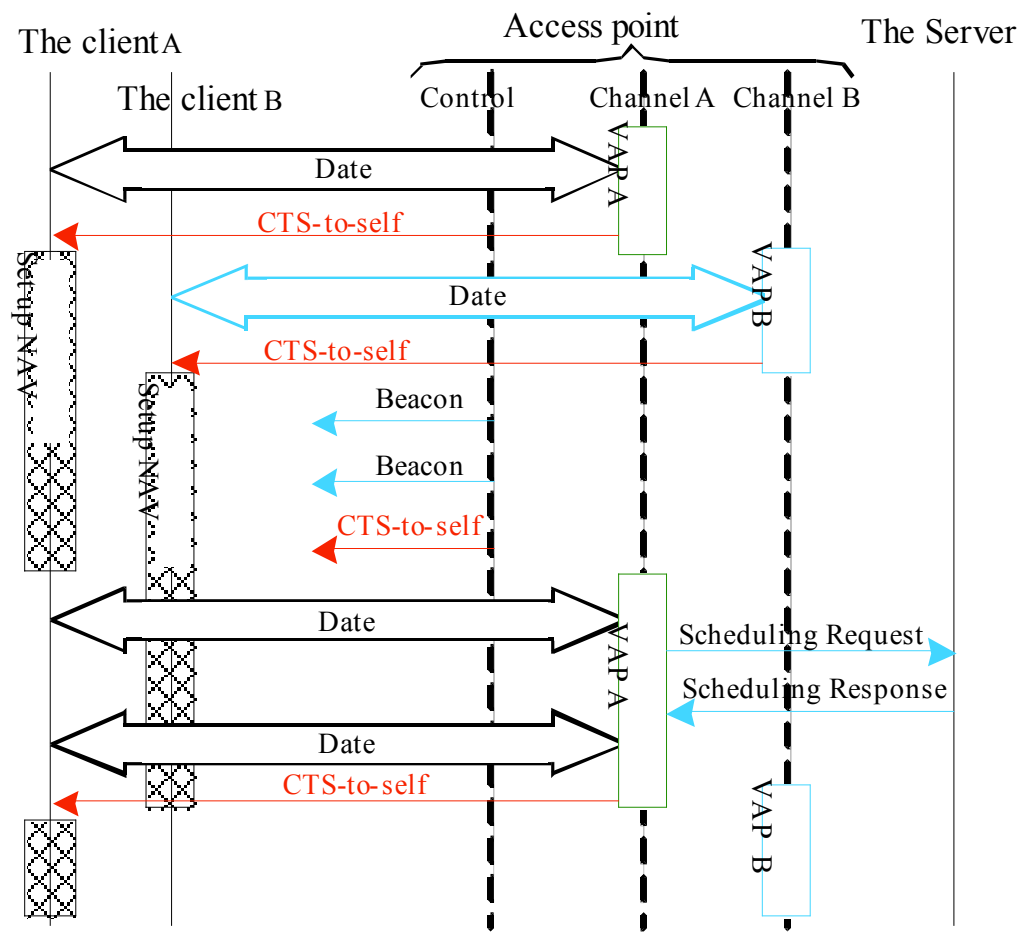

Fig. (7). The protecting mechanism of the client side when the access points switch different channels.

channel distributing rate of the present physical access points should be carried in the requests. After the servers is determined by the resource distribution algorithm, the Scheduling Response agrees the frequency using efficiency of virtual access points to changed or modified. When there are many data be transmitted in the virtual access point $\mathrm{A}$ and the client side $\mathrm{A}$, the virtual access point $\mathrm{A}$ can increase the time of delaying in the channel $\mathrm{A}$, as shown in the Fig. (7).

\section{THE HAND-OVER AND THE MOVING VIRTUAL ACCESS POINTS}

When the signals of the access points removed from the client side cover the ranges, the client side should do the channel scanning and search for the available access points. Later, the authentication, connection, and the data transmission can delay several seconds so that these procedures can have a greater kill-ability to the real-time network service. How the central service makes the client side seamlessly connect with other physical access points through the copy and the movement of the virtual access points is discussed in the paper.

The Fig. (8) shows that how the central service makes the moving virtual access points reach to the client side's seamless hand-over. If a certain client side has established the special virtual access points in the physical access point $A$, the upload and the download of the data flow can be maintained. The access points A can find out whether the client side can be far away with the signal coverage range of the access points through the signal strength supervision of the data frame or the ACK frame. In addition, the access points can also give the periodic measurement return to the client side so that the accuracy of the signal strength supervision can be strengthened without the transmission of the upload and the download data, in which the measurement type 2 and the type 7 can respectively return back the PRI and the frame error rate for judging whether the client side is locate in the border of the signal coverage rate. When the access point $\mathrm{A}$ judges that the client side A may leave the signal coverage range, the access point A can send the VAP Move Request to the virtual access points with the state of the present client side and the virtual access points to the client side, such as the state of having sent those frames, waiting for the confirming reply and the possible moving neighboring access. After the central server receives the VAP Move Request of the virtual access points, the server can send the VAP Duplication to the neighboring physical access points through the proper algorithm judgment. The information includes the related data and settings of the access points, containing the state of the related data in the previous physical access point A. After the access point $B$ receives the duplicated information of the virtual access points from the servers, its contents can duplicate the same virtual access points in the access point $\mathrm{A}$, even including the MAC address so that the client side cannot distinct the difference.

The access point $\mathrm{B}$ establishes the mirrored virtual access point aiming to the features of the wireless environment. Apart from receiving the data uploaded by the client side in the access point $\mathrm{A}$, the access point $\mathrm{B}$ can also receive the data uploaded by the client side so that the client side can share the benefits of the data backup in the weak signal border area. As to the repeated data, the upper layer (such as the TCP layer) can be automatically removed. Similarly, the downloading packets can be sent by the access point $\mathrm{A}$ and the access point $\mathrm{B}$, and the client side can remain the received packets. 


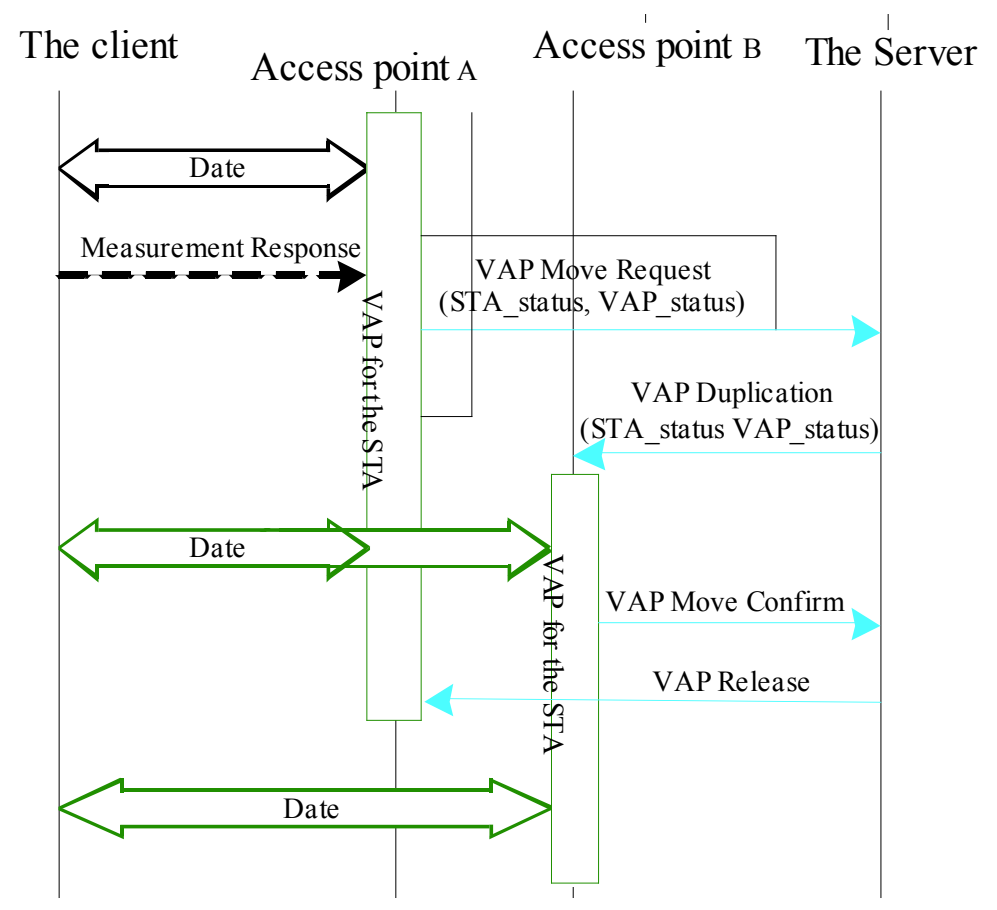

Fig. (8). The central service makes the client side seamlessly connect with other physical access points through the copy and the movement of the virtual access points.

Distributed through the establishment of the virtual access points so that the load balance can be optimal. The server can reduce the packet collision and interruption caused by the access points share with the channels and reduce the problems of the network speed down through the management of the virtual access points and the channels distributions. The servers can make the client side connect with the same access points without conducting the traditional hand-over procedure which can make the online interrupt by moving the mechanism of the virtual access points.

At present, the software-defined wireless access network is still in the developing phase, and many opening issues are still not obtaining the proper solutions. In the future, some issues will be attempted for offering a more accurate solution.

\section{CONFLICT OF INTEREST}

The authors confirm that this article content has no conflict of interest.

\section{ACKNOWLEDGEMENTS}

Declared none.

\section{REFERENCES}

[1] METIS Project Document No. ICT-317669-METIS/D1.1. Scenarios, requirements and KPIs for $5 \mathrm{G}$ mobile and wireless system, 2013.

[2] Part 11: Wireless LAN Medium Access Control (MAC) and Physical Layer (PHY) Specifications, 1999.

[3] R. Zhang, and S. Cui, "Cooperative Interference Management With MISO Beamforming," IEEE Transactions on Signal Processing, vol. 58 , pp. 5450-5458, 2010.
[4] W. D. Lin and H. Y. Hsieh, "Joint optimization of resource allocation and modulation coding schemes for unicast video streaming in OFDMA networks," In: IEEE PIMRC, 2012.

[5] Y. E. Lin, K. H. Liu, and H. Y. Hsieh, "Design of power control protocols for spectrum sharing in cognitive radio networks: a gametheoretic perspective," In: IEEE ICC, pp. 1-6, 2010.

[6] A. A. Bertossi, C.M. Pinotti and R.B. Tan, "Channel assignment with separation for interference avoidance in wireless networks," IEEE Transactions on Parallel and Distributed Systems, pp. 222235, 2003.

[7] C. Poongodi, and A. Shanmugam, "Design and performance analysis of side by side, echelon and $\mathrm{H}$-shaped multiple printed dipole antennas for wireless local area network application," International Journal of Communication Systems, vol. 27, no. 12, pp 3781-3790, 2014.

[8] K. P. Bagadi, "Neural network-based multiuser detection for SDMA-OFDM system over IEEE $802.11 \mathrm{n}$ indoor wireless local area network channel models," International Journal of Electronics, vol. 100, no. 10, pp 1332-1347, 2013.

[9] M. Mahdi, "Small planar dual-band microstrip-fed monopole antenna for wireless local area network applications using slotted conductor-backed plane," Microwave and Optical Technology Letters, vol. 55, no. 10, pp. 2380-2383, 2013.

[10] Y. Zhou, Z. Luo, and H. Zhuang, "Sensor-assisted coverage selfoptimization for wireless local area network, " In: Proceedings 2013 Wireless and Optical Communications Conference, WOCC, 2013, pp. 444-448.

[11] M. Wei, "Passive wireless local area network radar network using compressive sensing technique" IET Radar, Sonar and Navigation, vol. 9 , no. 1 , pp. 84-91, 2015.

[12] X. Hu, "Study on marine automatic identification system based on wireless local area network," Applied Mechanics and Materials, Mechanical Engineering, Industrial Electronics and Informatization, vol. 299, pp. 156-159, 2013.

[13] B. Sikdar, "A study of the environmental impact of wired and wireless local area network access," IEEE Transactions on Consumer Electronics, vol. 59, no. 1, pp. 85-92, 2013.

[14] U. Singh, and P. Jindal, "Impact of transmission power on the performance of secure wireless local area," In: SCES Inspiring Engineering and Systems for Global Sustainability, 2014. 
[15] S. S. Karimabadi, and A. R. Attari, "Miniaturized coplanar waveguide-fed disk monopole antenna for ultra-wideband applications with improved wireless local area network band rejection," In- ternational Journal of RF and Microwave Computer-Aided Engineering, vol. 23, no. 1, pp. 83-89, 2013.

Received: June 10, 2015

Revised: July 29, 2015

Accepted: August 15, 2015

(C) Chen et al.; Licensee Bentham Open.

This is an open access article licensed under the terms of the (https://creativecommons.org/licenses/by/4.0/legalcode), which permits unrestricted, noncommercial use, distribution and reproduction in any medium, provided the work is properly cited. 